\title{
Review of: "Oxidized LDL-dependent pathway as new pathogenic trigger in arrhythmogenic cardiomyopathy"
}

jihong Han

Potential competing interests: The author(s) declared that no potential competing interests exist.

This paper investigates the role of oxidized low-density lipoprotein (oxLDL)-dependent activation of PPARg in the pathogenesis of arrhythmogenic cardiomyopathy (ACM). They found that oxLDL increased lipid accumulation in ACM patient-derived cardiac cells through PPARg-CD36 pathway. Then they confirmed these findings in mice. However, there are several limitations as following:

1. Figures $6 \mathrm{~A}$ and $\mathrm{B}$ do not seem to correspond to the legend, which states the plasma total cholesterol concentrations of WT and PKP2 ${ }^{+/-}$mice fed CDs or 3 months of HFD, while there are only two lines corresponding to $\mathrm{CD}$ and HFD (Figure $6 \mathrm{~A}$ ) and two groups (Figure 6B) in the figures, which do not show genotypes. It seems unclear if the author means the sum of the blood cholesterol levels of the two genotypes under each feeding method. The data is not presented completely enough, and the description is not clear enough.

2. The legend in Figure $6 C$ "For comparison, quantification of ORO positive area of cardiac sections of CDfed WT and Pkp2+/- mice ( $n=9$; two-way ANOVA) is shown (Appendix Fig S3)." is incorrect. It is shown in Figure S6.

3. The images and statistics are inconsistent in Figures 6D and S6B. It will be better to include the two genotype mice fed by CD in Figure 6.

4. There is a similar problem as the above in Figure 7. There are only two groups corresponding to HFD or HFD plus atorvastatin (Figure 7A and B), which do not show genotypes. No images of HFD-fed PKP2 ${ }^{+/-}$ mice without atorvastatin administration were given in Figure 7.

5. The number of patients recruited in this study does not rule out the possibility of being too small. The authors did not provide a sample size selection basis for this type of clinical data analysis, so it may be likely that the relationship between oxLDL and ACM phenotypes is highly accidental and exaggerated.

6. Experiments with CD36 and PPARg knockdown or knockout may be needed in the in vivo study to support the conclusion. 